\title{
Staged partial hepatectomy versus transarterial chemoembolization for the treatment of spontaneous hepatocellular carcinoma rupture: a multicenter analysis in Korea
}

\author{
Hyung Soon Lee', Gi Hong Choi'2, Jin Sub Choi'2, Kwang-Hyub Han ${ }^{3}$, Sang Hoon Ahn³, Do Young Kim³, \\ Jun Yong Park ${ }^{3}$, Seung Up Kim${ }^{3}$, Sung Hoon $\mathrm{Kim}^{4}$, Dong Sup Yoon ${ }^{5}$, Jae Keun Kim${ }^{5}$, Jong Won Choi \\ Soon Sun $\mathrm{Kim}^{7}$, Hana Park ${ }^{8}$ \\ ${ }^{1}$ Department of Surgery, National Health Insurance Service I/san Hospital, Goyang, Korea \\ ${ }^{2}$ Department of Surgery, Severance Hospital, Yonsei University College of Medicine, Seoul, Korea \\ ${ }^{3}$ Department of Internal Medicine, Severance Hospital, Yonsei University College of Medicine, Seoul, Korea \\ ${ }^{4}$ Department of Surgery, Wonju Severance Christian Hospital, Yonsei University Wonju College of Medicine, Wonju, Korea \\ ${ }^{5}$ Department of Surgery, Gangnam Severance Hospital, Yonsei University College of Medicine, Seoul, Korea \\ ${ }^{6}$ Department of Internal Medicine, National Health Insurance Service I/san Hospital, Goyang, Korea \\ 'Department of Internal Medicine, Ajou University Medical Center, Ajou University School of Medicine, Suwon, Korea \\ ${ }^{8}$ Department of Internal Medicine, CHA Bundang Medical Center, CHA University, Seongnam, Korea
}

\begin{abstract}
Purpose: The aim of this study was to identify the prognostic factors and compare the long-term outcomes of staged hepatectomy and transarterial chemoembolization (TACE) for patients with spontaneous rupture of hepatocellular carcinoma (HCC).

Methods: This study is a multicenter, retrospective analysis of patients with newly diagnosed ruptured HCC. To compare overall survival between staged hepatectomy group and TACE alone group, we performed propensity score-matching to adjust for significant differences in patient characteristics. To identify prognostic factors, the clinical characteristics at the time of diagnosis of tumor rupture were investigated using Cox-regression analysis.

Results: From 2000 to 2014, 172 consecutive patients with newly diagnosed ruptured HCC were treated in 6 Korean centers. One hundred seventeen patients with Child-Pugh class A disease were identified; of which 112 were initially treated with transcatheter arterial embolization (TAE) for hemostasis and five underwent emergency surgery for bleeder ligation. Of the 112 patients treated with TAE, 44 underwent staged hepatectomy, 61 received TACE alone, and 7 received conservative treatment after TAE. Those that underwent staged hepatectomy had significantly higher overall survival than those that underwent TACE alone before matching $(P<0.001)$ and after propensity score-matching $(P=0.006)$. Multivariate analysis showed that type of treatment, presence of portal vein thrombosis, pretreatment transfusion $>1,200$ $\mathrm{mL}$, and tumor size $>5 \mathrm{~cm}$ were associated with poor overall survival.

Conclusion: Staged hepatectomy may offer better long-term survival than TACE alone for spontaneous rupture of HCC. Staged hepatectomy should be considered in spontaneous rupture of HCC with resectable tumor and preserved liver function.

[Ann Surg Treat Res 2019;96(6):275-282]
\end{abstract}

Key Words: Hepatocellular carcinoma, Spontaneous rupture, Hepatectomy, Therapeutic chemoembolization

Received December 21, 2018, Revised March 16, 2019,

Accepted April 1, 2019

Corresponding Author: Jin Sub Choi

Department of Surgery, Severance Hospital, Yonsei University College of

Medicine, 50-1 Yonsei-ro, Seodaemoon-gu, Seoul 03722, Korea

Tel: +82-2-2228-2122, Fax: +82-2-313-8289

E-mail: choi5491@yuhs.ac

ORCID code: https://orcid.org/0000-0002-6467-6494
Copyright (c) 2019, the Korean Surgical Society

(c) Annals of Surgical Treatment and Research is an Open Access Journal. All articles are distributed under the terms of the Creative Commons Attribution NonCommercial License (http://creativecommons.org/licenses/by-nc/4.0/) which permits unrestricted non-commercial use, distribution, and reproduction in any medium, provided the original work is properly cited. 


\section{INTRODUCTION}

Spontaneous rupture of hepatocellular carcinoma (HCC), a life-threatening complication with a high mortality rate (25\%-75\%), develops in about $2 \%-12 \%$ of patients with HCC [1-3]. Previous studies have reported that the prognosis of ruptured HCC is very poor, and that spontaneous tumor rupture occurs at an advanced tumor stage in patients with a poor liver functional reserve [4]. And the current TNM staging systems still classify ruptured HCC as T4 [5].

According to recent studies, the classification of all ruptured HCC as T4 may not be reliable $[2,3,6]$. A nationwide survey in Japan demonstrated that tumor rupture itself had a negative impact on patient survival [2]; however, the negative impact of spontaneous tumor rupture only corresponded to an additional 0.5 to $2.0 \mathrm{TNM}$ stages on the baseline tumor status, which was not strong enough to cancel the effects of other tumor-related parameters. Additionally, Chan et al. [3] recently reported that HCC rupture appeared to increase the T-stage by one level for otherwise T1-2 tumors but had no additional prognostic impact on T3 tumors or on tumors $>10 \mathrm{~cm}$. Thus, classifying all ruptured HCC as T4 may not reliably predict long-term outcomes, and the significance of tumor rupture in the tumor staging system has been so far been only provisional.

Actually, spontaneous ruptured HCCs are heterogeneous, with different liver functional statuses and various tumor stages, excluding the rupture factor. Thus, although the patients had a recent rupture episode, the treatment is crucial for the long-term outcomes, allowing for liver functional reserve and resectable tumor status. Resection and transarterial chemoembolization (TACE) are widely performed for ruptured HCC. Although many previous studies reported favorable longterm outcomes of staged partial hepatectomy, the optimal treatment for ruptured HCC remains under debate $[3,6,7]$. Additionally, it remains uncertain whether TACE actually has therapeutic benefits for patients with spontaneous rupture of HCC [8-10].

Thus, we aimed to identify the prognostic factors and to compare the long-term outcomes of staged hepatectomy and TACE for patients with spontaneous ruptured HCC.

\section{METHODS}

\section{Patient selection}

We retrospectively collected data of patients managed for spontaneous rupture of HCC during the period from January 2000 to December 2014 in 6 Korean centers. The diagnosis of spontaneous rupture of HCC was confirmed using dynamic contrast-enhanced abdominal CT [11]. The medical records were retrospectively reviewed after approval by the Institutional Review Boards of Yonsei University College of Medicine and the requirement for informed consent was waived (4-2014-0912).

\section{Review of patient data}

We analyzed clinical data including age, sex, hepatitis B virus surface antigen positivity, Child-Pugh class, shock on presentation, administration of blood transfusions, serum $\alpha$-FP and des-gamma-carboxy prothrombin (DCP), hemoglobin, platelet count, creatinine, aspartate aminotransferase, alanine aminotransferase, total bilirubin, international normalized ratio, number of hospitalization days, type of treatment, time from HCC rupture until surgery, type of surgery, complications of surgery, recurrence pattern after surgery, duration of followup, and outcome. Only the last measurements of $\alpha$-FP and DCP prior to treatment were considered. Major hepatectomy was defined as the resection of $\geq 3$ anatomical segments, as described by Couinaud. Imaging features were determined by pretreatment radiologic findings including tumor multiplicity, size, and location and presence of portal vein thrombosis.

\section{Treatment and patient follow-up}

In hemodynamically unstable patients with continuous hemorrhage, transcatheter arterial embolization (TAE) was the first intervention. If TAE failed, emergency laparotomy was performed. After the hemodynamic status was stabilized, the patients underwent complete clinical evaluation to formulate a definitive HCC treatment plan, including serology, Child-Pugh grading, abdominal $\mathrm{CT}$, hepatic angiography, and measurement of indocyanine green retention at 15 minutes. The feasibility of hepatectomy was assessed based on the test results. In surgical candidates, elective hepatectomy was preformed, with the resection type decided based on the liver functional reserve and patient performance status [6]. In patients for whom surgery was not an option, TACE was performed 2-4 months after TAE. All patients were followed up at least every 3 months. Dynamic CT was performed at each follow-up appointment, and serum $\alpha$-FP and DCP levels were screened. HCC recurrence was defined as newly developed lesions detected by CT. Patients with suspected recurrence underwent magnetic resonance imaging or hepatic angiography.

\section{Analysis of prognosis and treatment outcome}

We compared the survival curves of the patients that received staged hepatectomy or TACE alone to evaluate the impacts of the treatments on patient prognosis. To identify prognostic factors for patients with spontaneous ruptured HCC, we investigated the clinical characteristics at the time of diagnosis of tumor rupture using Cox-regression analysis. The analysis of postoperative complications employed the Clavien-Dindo classification [12]. Pleural effusion, ileus, and fluid accumulation in the operating field were radiologically diagnosed. Acute renal failure was defined as abrupt and reversible declines in renal 
filtration function.

To compare treatment outcomes between staged hepatectomy and TACE alone in spontaneously ruptured HCC, we performed propensity score-matching to adjust for significant differences in patient characteristics. We excluded matching the patients with concomitant extrahepatic metastasis at diagnosis of HCC. Thus, we conducted propensity score-matching on the remaining 85 ruptured HCC patients who underwent TACE alone to select the matched group for staged hepatectomy group.

\section{Statistical analysis}

The propensity scores were estimated by multiple logistic regression analysis. Regression analysis predicted the probability that each patient would be treated based on 5 covariables: age, hemoglobin, tumor size, multiple tumor, and Child-Pugh grade. Using these 5 covariables in the regression analysis, a propensity score was calculated for each patient. The discrimination and calibration abilities of the propensity score models were assessed with the C-statistic and the HosmerLemeshow statistic [6]. The model was then used to obtain a one-to-one match for the staged hepatectomy group and TACE alone group. Finally, each patient with staged hepatectomy was matched to one patient with TACE alone, and the identical propensity score. After the propensity score-matched sample was formed, we assessed baseline variable balance between the 2 propensity-matched cohort groups. Continuous variables were compared with the paired t-test or the Wilcoxon signed rank test, as appropriate, and categorical variables were compared with the McNemar's or marginal homogeneity test, as appropriate [13]. Propensity score-matching were performed with SAS ver. 9.2 (SAS Institute Inc, Cary, NC, USA).

Data are presented as the number of patients (percentage) or as the median value (range). We analyzed categorical variables using the chi-square test or Fisher exact test and continuous variables using the Mann-Whitney U-test. We calculated overall survival (OS) rates using the Kaplan-Meier method and evaluated differences in survival between groups using the log- rank test. We calculated OS as the time from treatment until death from any cause, or until the end of follow-up. Variables found to be significant prognostic factors by univariate analysis were considered in a multivariate analysis. We considered P-values $<0.05$ statistically significant. Statistical procedures were conducted with the IBM SPSS ver. 18.0 (IBM Co., Armonk, NY, USA).

\section{RESULTS}

\section{Patient characteristics}

A total of 172 patients with newly diagnosed ruptured HCC were treated in 6 Korean centers. The median followup duration among all patients was 5 months (range, 0-162 months). The most frequently performed treatment was TACE (52.3\%), followed by surgery (28.5\%) and supportive care (13.4\%) (Table 1). Chemotherapy and radiotherapy were each performed in 5 of patients (2.9\%). The patients were managed according to the flow chart in Fig. 1. Eight patients had concomitant extrahepatic metastasis, and 117 patients had Child-Pugh class A disease. Among the 117 patients initially treated with TAE for hemostasis, 49 underwent surgery, 61 received TACE alone, and 7 received conservative treatment after TAE. Among the 49 patients that underwent surgery, 44 underwent staged hepatectomy after TAE, and 5 underwent emergency laparotomy due to hemostasis failure after TAE (bleeder ligation in 4 patients, tumor enucleation in 1 patient).

Among the 44 patients that underwent staged hepatectomy, 22 underwent major hepatectomy (11 right hepatectomy, 7 left hepatectomy, 2 left extended hepatectomy, and 2 central bisectionectomy) and 22 underwent minor hepatectomy ( 6 lateral sectionectomy, 1 anterior sectionectomy, 1 posterior sectionectomy, 8 segmentectomy, and 6 wedge resection). The median time between TAE and staged hepatectomy was 16 days (range, 7-375 days).

The patient characteristics at the time of HCC rupture diagnosis are summarized in Table 2. Compared with the patients that received staged hepatectomy, the patients that

Table 1. Patient numbers according to the treatment modality and center

\begin{tabular}{|c|c|c|c|c|c|c|}
\hline Center & Total, n (\%) & Surgery & TACE & $\begin{array}{l}\text { Supportive } \\
\text { care }\end{array}$ & $\begin{array}{l}\text { Chemo- } \\
\text { therapy }\end{array}$ & $\begin{array}{l}\text { Radio- } \\
\text { therapy }\end{array}$ \\
\hline Severance Hospital & $60(34.9)$ & 27 & 31 & 2 & 0 & 0 \\
\hline Gangnam Severance Hospital & $25(14.5)$ & 6 & 11 & 3 & 2 & 3 \\
\hline Wonju Severance Christian Hospital & $32(18.6)$ & 7 & 17 & 7 & 1 & 0 \\
\hline $\mathrm{CHA}$ Medical Center & $15(8.7)$ & 2 & 12 & 1 & 0 & 0 \\
\hline National Health Insurance Service Ilsan Hospital & $20(11.6)$ & 2 & 8 & 10 & 0 & 0 \\
\hline Ajou University Medical Center & $20(11.6)$ & 5 & 11 & 0 & 2 & 2 \\
\hline Total & $172(100)$ & $49(28.5)$ & $90(52.3)$ & $23(13.4)$ & $5(2.9)$ & $5(2.9)$ \\
\hline
\end{tabular}

TACE, transarterial chemoembolization. 


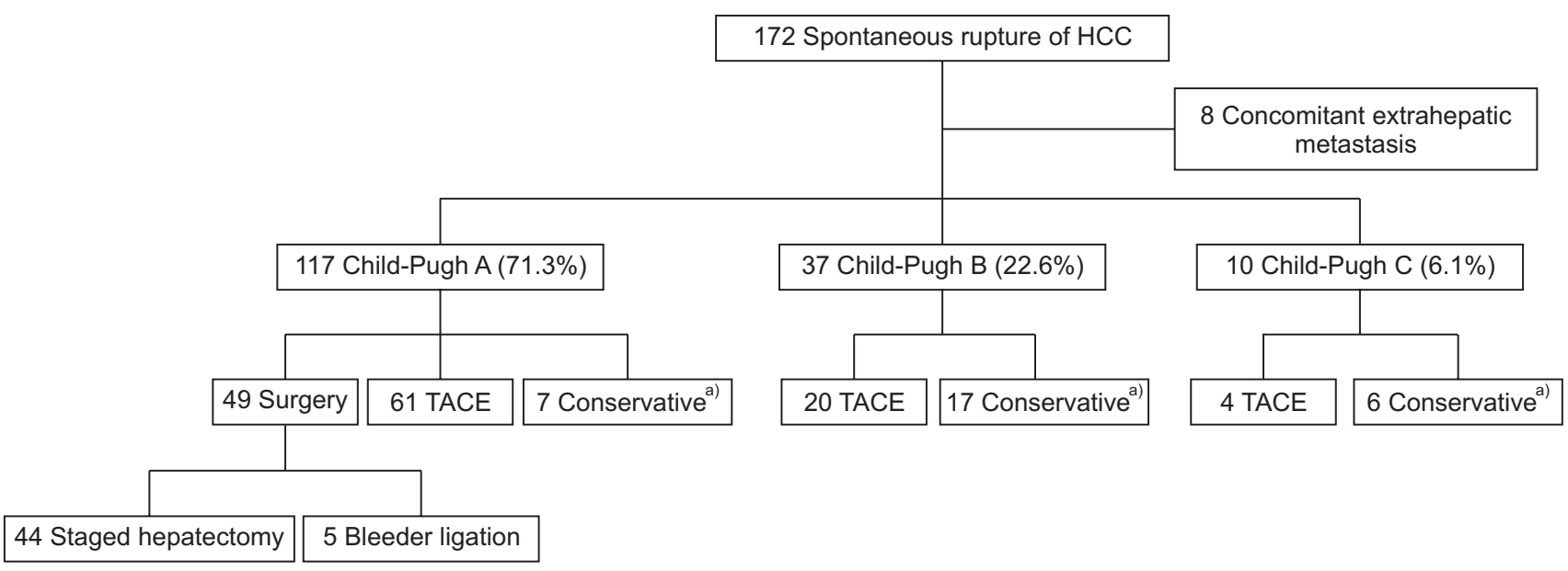

Fig. 1. Patient selection schematic. HCC, hepatocellular carcinoma; TACE, transarterial chemoembolization. ${ }^{\text {a) }}$ Supportive care, radiotherapy and chemotherapy.

Table 2. Patient characteristics at the time of diagnosis of tumor rupture

\begin{tabular}{|c|c|c|c|}
\hline Characteristic & Staged hepatectomy $(n=44)$ & TACE alone $(n=61)$ & P-value \\
\hline Age $(y r)$ & $51.5(18-80)$ & $61(32-79)$ & 0.009 \\
\hline Male sex & $34(77.3)$ & $52(85.2)$ & 0.295 \\
\hline HBV surface antigen $(+)$ & $36(81.8)$ & $42(68.9)$ & 0.495 \\
\hline Shock on presentation & $13(29.5)$ & $24(39.3)$ & 0.300 \\
\hline Portal vein thrombosis & $4(9.1)$ & $12(19.7)$ & 0.137 \\
\hline Tumor size $(\mathrm{cm})$ & $6.7(2.2-15.0)$ & $8.4(2.9-20.0)$ & 0.012 \\
\hline Multiple tumor & $5(11.4)$ & $26(42.6)$ & 0.001 \\
\hline Pretreatment transfusion & $21(47.7)$ & $37(60.7)$ & 0.189 \\
\hline Pretreatment transfusion $>1,200(\mathrm{~mL})$ & $12(27.3)$ & $24(39.3)$ & 0.199 \\
\hline$\alpha-\mathrm{FP}(\mathrm{ng} / \mathrm{mL})$ & $144.5(1.1-83,000)$ & $62(1.8-200,000)$ & 0.082 \\
\hline Des-gamma-carboxy prothrombin $(\mathrm{AU} / \mathrm{mL})$ & $806.5(13.0-23,641)$ & $2,000(20.0-69,660)$ & 0.249 \\
\hline Hemoglobin $(g / d L)$ & $11.6(8.0-16.2)$ & $10.7(5.5-15.9)$ & 0.045 \\
\hline Platelet $\left(10^{3} / \mu \mathrm{L}\right)$ & $184(69-467)$ & $165(65-454)$ & 0.138 \\
\hline Creatinine $(\mathrm{mg} / \mathrm{dL})$ & $0.8(0.3-3.0)$ & $1.0(0.5-4.1)$ & 0.419 \\
\hline Aspartate aminotransferase (IU/L) & $52(12-386)$ & $67(20-466)$ & 0.061 \\
\hline Alanine aminotransferase (IU/L) & $45.5(7-136)$ & $43(11-288)$ & 0.084 \\
\hline Total bilirubin (mg/dL) & $0.75(0.2-1.7)$ & $0.9(0.2-2.8)$ & 0.006 \\
\hline International normalized ratio & $1.06(0.34-1.61)$ & $1.13(0.84-1.77)$ & 0.078 \\
\hline
\end{tabular}

Values are presented as median (range) or number (\%).

TACE, transarterial chemoembolization.

received TACE alone had significantly lower hemoglobin levels and significantly greater age, tumor size, frequency of multiple tumors, and total bilirubin levels.

\section{Staged hepatectomy complications}

Seventeen patients that underwent staged hepatectomy (16.2\%) experienced complications. Grade I complications developed in nine patients (4 superficial surgical wound infection and 5 pleural effusion) and were managed conservatively. Grade II complications developed in eight patients $(5$ fluid accumulation at the resected liver surface, 2 postoperative ileus, and 1 acute renal failure). The patients with fluid accumulation were successfully treated with antibiotic optimization. The patients with ileus successfully recovered after nasogastric tube placement. The patient with renal failure received intermittent diuretic therapy and recovered normal renal filtration rates. There were no complications above type IIIa.

\section{Comparison of OS between staged hepatectomy and TACE alone}

The 1-, 3-, and 5-year OS rates were respectively $40.1 \%$, $15.7 \%$, and $10.5 \%$ in the patients that underwent TACE alone 

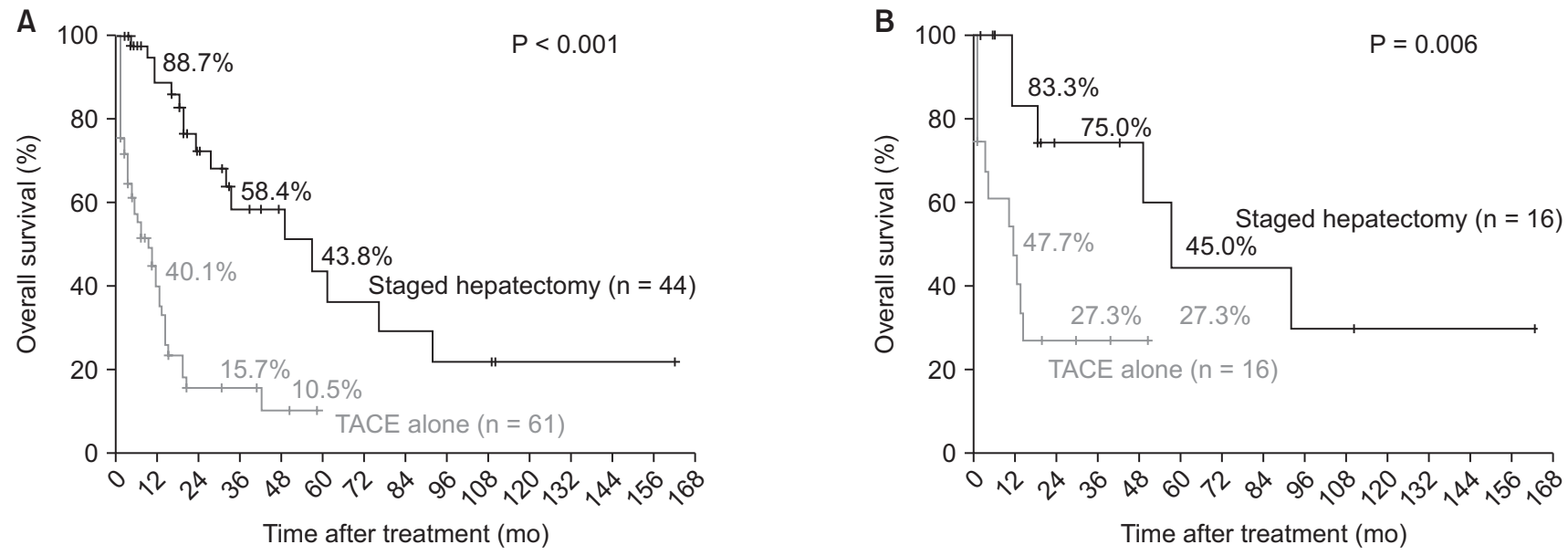

Fig. 2. Comparison of overall survival between staged hepatectomy and TACE alone before matching (A) and after propensity score-matching (B). TACE, transarterial chemoembolization.

Table 3. Before and after propensity score matching of baseline variables in the staged hepatectomy group versus the TACE alone group

\begin{tabular}{|c|c|c|c|c|c|c|}
\hline \multirow[b]{2}{*}{ Variable } & \multicolumn{3}{|c|}{ Before matching } & \multicolumn{3}{|c|}{ After matching } \\
\hline & $\begin{array}{l}\text { Staged hepatectomy } \\
\qquad(\mathrm{n}=44)\end{array}$ & $\begin{array}{l}\text { TACE alone } \\
\quad(\mathrm{n}=85)\end{array}$ & P-value & $\begin{array}{l}\text { Staged hepatectomy } \\
\qquad(\mathrm{n}=16)\end{array}$ & $\begin{array}{l}\text { TACE alone } \\
\quad(\mathrm{n}=16)\end{array}$ & P-value \\
\hline Age $(y r)$ & $53.43 \pm 12.70$ & $59.63 \pm 12.07$ & 0.008 & $57.38 \pm 13.64$ & $57.38 \pm 14.66$ & $>0.999$ \\
\hline Hemoglobin (g/dL) & $11.01 \pm 2.16$ & $10.55 \pm 2.27$ & 0.262 & $11.44 \pm 1.97$ & $11.35 \pm 1.73$ & 0.902 \\
\hline Tumor size $(\mathrm{cm})$ & $6.98 \pm 3.23$ & $9.25 \pm 4.51$ & 0.001 & $7.82 \pm 3.42$ & $7.83 \pm 2.63$ & 0.994 \\
\hline Multiple tumor & $5(11.4)$ & $38(44.7)$ & $<0.001$ & $2(12.5)$ & $2(12.5)$ & $>0.999$ \\
\hline \multicolumn{7}{|l|}{ Child-Pugh grade } \\
\hline A & $44(100)$ & $61(71.8)$ & $<0.001$ & $16(100)$ & $16(100)$ & $>0.999$ \\
\hline B & $0(0)$ & $20(23.5)$ & & $0(0)$ & $0(0)$ & \\
\hline $\mathrm{C}$ & $0(0)$ & $4(4.7)$ & & $0(0)$ & $0(0)$ & \\
\hline
\end{tabular}

Values are presented as mean \pm standard deviation or number $(\%)$.

TACE, transarterial chemoembolization.

and $88.7 \%, 58.4 \%$, and $43.8 \%$ in those that underwent staged hepatectomy (Fig. 2). The patients that underwent staged hepatectomy had significantly greater OS than those that underwent TACE alone $(\mathrm{P}<0.001)$.

\section{Comparison of OS between staged hepatectomy and TACE alone after propensity score matching} The 2 groups showed significant differences in age, tumor size, multiple tumor and Child-Pugh grading, before matching (Table 3). These differences between groups were eliminated after matching. After matching, 1-, 3-, and 5-year OS rates were, respectively, $47.7 \%, 27.3 \%$, and $27.3 \%$ in the TACE-alone group and $83.3 \%, 75.0 \%$, and $45.0 \%$ in the staged hepatectomy group (Fig. 2). After matching, staged hepatectomy group also had significantly greater OS than those that underwent TACE alone group $(\mathrm{P}=0.006)$.

\section{Prognostic factors for OS}

In the univariate analysis, portal vein thrombosis, type of treatment, pretreatment transfusion $>1,200 \mathrm{~mL}$, serum $\alpha-\mathrm{FP}$ level, tumor size $>5 \mathrm{~cm}$, and multiple tumors were significantly associated with OS (Table 4). In the multivariate analysis, type of treatment, presence of portal vein thrombosis, pretreatment transfusion $>1,200 \mathrm{~mL}$, and tumor size $>5 \mathrm{~cm}$ were associated with poor OS.

\section{Recurrence pattern after staged hepatectomy}

$\mathrm{HCC}$ recurrence developed in 31 patients that underwent staged hepatectomy $(70.5 \%)$. Common sites of HCC recurrence after staged hepatectomy were the liver ( $n=16 ; 51.6 \%$ ), lung ( $n=7 ; 22.6 \%)$, peritoneum $(n=4 ; 12.9 \%)$, lymph nodes $(n=2$; $6.5 \%)$, adrenal gland $(\mathrm{n}=1 ; 3.2 \%)$, and spleen $(\mathrm{n}=1 ; 3.2 \%)$. 
Table 4. Cox-regression analysis of prognostic factors for overall survival

\begin{tabular}{|c|c|c|c|}
\hline \multirow{2}{*}{ Variable } & \multicolumn{3}{|c|}{ Overall survival } \\
\hline & $\mathrm{HR}$ & $95 \% \mathrm{Cl}$ & P-value \\
\hline \multicolumn{4}{|l|}{ Univariate analyses } \\
\hline Age, 1-yr increase & 1.940 & $0.994-1.035$ & 0.164 \\
\hline Male sex & 0.008 & $0.516-1.829$ & 0.929 \\
\hline $\begin{array}{l}\text { HBV surface antigen positivity } \\
\text { No vs. yes }\end{array}$ & 1.095 & $0.367-1.356$ & 0.295 \\
\hline $\begin{array}{l}\text { Shock on presentation } \\
\text { No vs. yes }\end{array}$ & 0.140 & $0.531-1.537$ & 0.708 \\
\hline $\begin{array}{l}\text { Portal vein thrombosis } \\
\text { No vs. yes }\end{array}$ & 13.333 & $0.155-0.570$ & $<0.001$ \\
\hline $\begin{array}{l}\text { Type of treatment } \\
\text { Staged hepatectomy vs. TACE alone }\end{array}$ & 26.205 & $0.106-0.367$ & $<0.001$ \\
\hline Treatment institution & 0.391 & $0.901-1.223$ & 0.532 \\
\hline $\begin{array}{l}\text { Pretreatment transfusion }>1,200 \mathrm{~mL} \\
\text { No vs. yes }\end{array}$ & 5.005 & $0.319-0.927$ & 0.025 \\
\hline Hemoglobin $(g / d L)$ & 1.271 & $0.821-1.055$ & 0.260 \\
\hline Creatinine (mg/dL) & 2.109 & $0.371-1.159$ & 0.146 \\
\hline Albumin $(\mathrm{g} / \mathrm{dL})$ & 0.776 & $0.483-1.318$ & 0.378 \\
\hline Aspartate aminotransferase (IU/L) & 2.225 & $0.999-1.005$ & 0.136 \\
\hline Alanine aminotransferase (IU/L) & 0.697 & $0.997-1.007$ & 0.404 \\
\hline Platelet $\left(10^{3} / \mu \mathrm{L}\right)$ & 0.006 & $0.997-1.003$ & 0.940 \\
\hline Total bilirubin, mg/dL & 3.598 & $0.986-2.402$ & 0.058 \\
\hline International normalized ratio & 0.173 & $0.352-4.993$ & 0.678 \\
\hline$\alpha-\mathrm{FP}(\mathrm{ng} / \mathrm{mL})$ & 5.162 & $1.000-1.000$ & 0.023 \\
\hline Des-gamma-carboxy prothrombin (mAU/mL) & 0.415 & $1.000-1.000$ & 0.519 \\
\hline $\begin{array}{l}\text { Tumor size }>5 \mathrm{~cm} \\
\text { No vs. yes }\end{array}$ & 9.154 & $0.160-0.676$ & 0.002 \\
\hline $\begin{array}{l}\text { Multiple tumor } \\
\text { Solitary vs. multiple }\end{array}$ & 8.633 & $1.293-3.618$ & 0.003 \\
\hline Multivariate analysis & & & \\
\hline $\begin{array}{l}\text { Type of treatment } \\
\text { Staged hepatectomy vs. TACE alone }\end{array}$ & 17.027 & $0.126-0.478$ & $<0.001$ \\
\hline $\begin{array}{l}\text { Pre-treatment transfusion }>1,200 \mathrm{~mL} \\
\text { No vs. yes }\end{array}$ & 4.135 & $0.301-0.978$ & 0.042 \\
\hline $\begin{array}{l}\text { Portal vein thrombosis } \\
\text { No vs. yes }\end{array}$ & 10.485 & $0.143-0.620$ & 0.001 \\
\hline $\begin{array}{l}\text { Tumor size }>5 \mathrm{~cm} \\
\text { No vs. yes }\end{array}$ & 4.183 & $0.196-0.966$ & 0.041 \\
\hline
\end{tabular}

$\mathrm{HR}$, hazard ratio; $\mathrm{Cl}$, confidence interval; TACE, Transarterial chemoembolization.

\section{DISCUSSION}

Staged hepatectomy resulted in better long-term survival than TACE alone in patients with HCC with recent tumor rupture. There were no complications above type IIIa after staged hepatectomy. Multivariate Cox-regression analysis showed that the type of treatment, pretreatment transfusion $>1,200 \mathrm{~mL}$, presence of portal vein thrombosis, and tumor size $>5 \mathrm{~cm}$ were associated with poor prognosis in patients with spontaneous ruptured HCC. Those results suggest that the type of treatment may be an important factor for long-term survival in patients with ruptured HCC.
The treatment of spontaneous ruptured HCC remains a complicated issue, because patients often present with an advanced tumor stage and poor liver function. There is insufficient time to evaluate precise liver functional reserve and tumor status in such patients, because hemorrhage from ruptured HCC leads to coagulopathy and hemodynamic instability. Therefore, hemostasis is the primary purpose of initial treatment. Emergency hepatectomy has the advantage of achieving both hemostasis and definitive treatment in a single operation; however, it may be associated with high morbidity and mortality $[14,15]$. TAE is reported to be a highly effective first-line approach to achieving hemostasis during the acute 
phase of ruptured HCC, with the most recent reports citing a hemostasis success rate $>90 \%[8,9,16]$. Additionally, TAE allowed time to complete preoperative clinical evaluation of the tumor and liver functional status by successfully controlling bleeding, which facilitated selection of surgical candidates among patients with ruptured HCC. In the current study, there were no complications above type IIIa after staged hepatectomy. With regard to the low complication rates in our study, the full preoperative clinical evaluations after hemodynamic stabilization may contribute to low morbidity and mortality [6]. Thus, TAE facilitates full preoperative clinical evaluation, thereby allowing safe surgery in patients with ruptured HCC.

Because ruptured tumors are considered to be T4, many institutions tend to exclude curative treatment for spontaneous HCC rupture $[2,5,10,17]$. In the current staging paradigm, HCC rupture is classified as T4 disease regardless of other tumor characteristics. That classification has been questioned in some previous studies, which suggested that classifying all ruptured HCCs as T4 may not accurately reflect the true prognosis [2,3]. Our previous report also demonstrated that most resectable ruptured HCCs are small or medium-sized solitary tumors, and there were no T4 tumors among the ruptured HCCs treated with staged hepatectomy when the factor of tumor rupture was excluded from the classification [6]. Thus, ruptured HCC should not be considered a "singular clinical entity" with the rupture event as the sole driving prognostic factor [18].

To date, the largest clinical experience of ruptured HCC was reported by Aoki et al. [2] from a nationwide survey in Japan that evaluated 1,160 patients treated in 800 centers across the country with a 5 -year OS rate of $13.3 \%$. In that study, an analysis of OS stratified according to the main treatment modality showed that the 1-, 3-, and 5-year OS rates after hepatic resection were $76.0 \%, 48.6 \%$, and $33.9 \%$, respectively, and the 1 -, 3-, and 5-year OS rates after TACE were $39.7 \%, 14.1 \%$, and $6.0 \%$, respectively. A recent systematic review showed that when OS was stratified by treatment modality, TACE was associated with worse 1-year and 3-year OS (37.3\% and 17.3\%, respectively) than staged hepatectomy $(75.7 \%$ and $43 \%$, respectively) [18]. Those findings are consistent with our observations that patients that underwent TACE alone had significantly lower OS rates than those that underwent staged hepatectomy. Our results also suggest that, in selected cases, long-term survival can be expected if the patient's condition is tolerable regarding the curative treatment. Consequently, in patients with preserved liver function and resectable tumors, staged hepatectomy should be considered as a potential approach for achieving tumor control and long-term survival. TACE may be best employed in patients that are ineligible for surgery because of poor performance status, advanced underlying hepatic disease, or other comorbidities.

Previous studies provided data on prognostic factors associ- ated with OS in patients with ruptured HCC. Several factors were associated with worse prognosis, including tumor size $>10 \mathrm{~cm}$, T4 ruptured HCC, presence of cirrhosis, higher ChildPugh class, lower hemoglobin, higher creatinine, higher $\alpha-\mathrm{FP}$, higher total bilirubin, portal vein invasion, protrusion $>30 \%$ from the liver surface, and shock at diagnosis [18]. Those findings are consistent with our results regarding prognostic factors associated with OS following HCC rupture; however, the finding that pretreatment transfusion $>1,200 \mathrm{~mL}$ is a prognostic factor is unique to our study. The association between allogenic blood transfusion and clinical outcomes for patients with HCC remains controversial $[19,20]$. Kwon et al. [21] demonstrated that allogenic blood transfusion has no influence on the long-term oncological outcome in HCC. Kuroda et al. [22] reported that perioperative blood transfusion did not influence the overall and disease-free survival rates in patients with HCC that underwent curative resection. However, a recent meta-analysis demonstrated that allogenic blood transfusion has a significantly deleterious effect on clinical outcomes and postoperative complications in patients with HCC [20]. Our results demonstrated that pretreatment transfusion $>1,200$ $\mathrm{mL}$ had a negative effect on OS in patients with ruptured HCC. In that regard, there is a need to minimize blood loss during the management of patients with spontaneous ruptured HCC, and meticulous bleeding control is essential during staged hepatectomy

This study has several limitations. Although it included multiple centers, it included only a small number of patients. Furthermore, the study was designed retrospectively, so selection bias is possible. And most of the patients had HBVrelated HCC, which is not the case in patient populations in Western countries [23].

In summary, our results indicate that staged partial hepatectomy may offer better long-term survival than TACE alone for patients with HCC with recent tumor rupture. Staged hepatectomy should be considered in cases of spontaneous HCC rupture with resectable tumor and preserved liver function. More specifically, staged hepatectomy should be considered in cases of absence of portal vein thrombosis, pretreatment transfusion $\leq 1,200 \mathrm{~mL}$, and tumor size $\leq 5 \mathrm{~cm}$. Furthermore, rupture itself may not be a prognostic factor for unfavorable surgical outcomes. Further clinical studies to determine the true impact of HCC rupture with regard to long-term prognosis are required in the form of prospective randomized trials with adequate sample sizes and prolonged follow-up.

\section{CONFLICTS OF INTEREST}

No potential conflict of interest relevant to this article was reported. 


\section{ACKNOWLEDGEMENTS}

This study was supported by a faculty research grant from

Yonsei University College of Medicine (6-2014-0169).

\section{REFERENCES}

1. Lai EC, Lau WY. Spontaneous rupture of hepatocellular carcinoma: a systematic review. Arch Surg 2006;141:191-8.

2. Aoki T, Kokudo N, Matsuyama Y, Izumi N, Ichida T, Kudo M, et al. Prognostic impact of spontaneous tumor rupture in patients with hepatocellular carcinoma: an analysis of 1160 cases from a nationwide survey. Ann Surg 2014:259:532-42.

3. Chan AC, Dai JW, Chok KS, Cheung TT, Lo CM. Prognostic influence of spontaneous tumor rupture on hepatocellular carcinoma after interval hepatectomy. Surgery 2016;159:409-17.

4. Liu CL, Fan ST, Lo CM, Tso WK, Poon RT, Lam CM, et al. Management of spontaneous rupture of hepatocellular carcinoma: single-center experience. J Clin Oncol 2001:19:3725-32.

5. Amin MB, Edge S, Greene F, Byrd DR, Brookland RK, Washington MK, et al. AJCC cancer staging manual. 8th ed. New York: Springer; 2017.

6. Lee HS, Choi GH, Kang DR, Han KH, Ahn SH, Kim DY, et al. Impact of spontaneous hepatocellular carcinoma rupture on recurrence pattern and long-term surgical outcomes after partial hepatectomy. World J Surg 2014:38:2070-8.

7. Yang T, Sun YF, Zhang J, Lau WY, Lai EC, Lu JH, et al. Partial hepatectomy for ruptured hepatocellular carcinoma. Br J Surg 2013;100:1071-9.

8. Kim JY, Lee JS, Oh DH, Yim YH, Lee HK. Transcatheter arterial chemoembolization confers survival benefit in patients with a spontaneously ruptured hepatocellular carcinoma. Eur J Gastroenterol Hepatol 2012;24:640-5.

9. Yoshida H, Mamada Y, Taniai N, Uchida E. Spontaneous ruptured hepatocellular carcinoma. Hepatol Res 2016;46:13-21.

10. Chan WH, Hung CF, Pan KT, Lui KW, Huang YT, Lin SY, et al. Impact of spontaneous tumor rupture on prognosis of patients with T4 hepatocellular carcinoma. J Surg Oncol 2016;113:789-95.

11. Zhu LX, Wang GS, Fan ST. Spontaneous rupture of hepatocellular carcinoma. $\mathrm{Br} \mathrm{J}$ Surg 1996;83:602-7.

12. Clavien PA, Barkun J, de Oliveira ML, Vauthey JN, Dindo D, Schulick RD, et al. The Clavien-Dindo classification of surgical complications: five-year experience. Ann Surg 2009:250:187-96.

13. Austin PC. A critical appraisal of propensity-score matching in the medical literature between 1996 and 2003. Stat Med 2008:27:2037-49.

14. Vergara V, Muratore A, Bouzari H, Polastri R, Ferrero A, Galatola G, et al. Spontaneous rupture of hepatocelluar carcinoma: surgical resection and long-term survival. Eur J Surg Oncol 2000;26:770-2.

15. Lai EC, Wu KM, Choi TK, Fan ST, Wong J. Spontaneous ruptured hepatocellular carcinoma. An appraisal of surgical treatment. Ann Surg 1989;210:24-8.

16. Monroe EJ, Kogut MJ, Ingraham CR, Kwan SW, Hippe DS, Padia SA. Outcomes of emergent embolisation of ruptured hepatocellular carcinoma in a western population. Clin Radiol 2015;70:730-5.

17. Han XJ, Su HY, Shao HB, Xu K. Prognostic factors of spontaneously ruptured hepatocellular carcinoma. World J Gastroenterol 2015;21:7488-94.

18. Moris D, Chakedis J, Sun SH, Spolverato G, Tsilimigras DI, Ntanasis-Stathopoulos I, et al. Management, outcomes, and prognostic factors of ruptured hepatocellular carcinoma: a systematic review. J Surg Oncol 2018;117:341-53.

19. You DD, Kim DG, Seo CH, Choi HJ, Yoo YK, Park YG. Prognostic factors after curative resection hepatocellular carcinoma and the surgeon's role. Ann Surg Treat Res 2017:93:252-9.

20. Liu L, Wang Z, Jiang S, Shao B, Liu J, Zhang $\mathrm{S}$, et al. Perioperative allogenenic blood transfusion is associated with worse clinical outcomes for hepatocellular carcinoma: a meta-analysis. PLoS One 2013;8:e64261.

21. Kwon AH, Matsui Y, Kamiyama Y. Perioperative blood transfusion in hepatocellular carcinomas: influence of immunologic profile and recurrence free survival. Cancer 2001;91:771-8.

22. Kuroda S, Tashiro H, Kobayashi T, Oshita A, Amano H, Ohdan $\mathrm{H}$. No impact of perioperative blood transfusion on recurrence of hepatocellular carcinoma after hepatectomy. World J Surg 2012;36:651-8.

23. Zhu RX, Seto WK, Lai CL, Yuen MF. Epidemiology of hepatocellular carcinoma in the Asia-Pacific region. Gut Liver 2016;10: 332-9. 